Clinical Case Report

\title{
Scrotal Pyocele in a Neonate: Review of the Literature and the Lessons Learned
}

\author{
Tina Lozej ${ }^{1}$, Darja Paro-Panjan ${ }^{2,3}$, Martin Thaler ${ }^{4}$, Robert Kordič ${ }^{5}$ Liza Lah ${ }^{6}$, Petja Fister ${ }^{2,3}$
}

${ }^{1}$ Department of Paediatrics, General Hospital Dr. Franc Derganc Nova Gorica, Slovenia, ${ }^{2}$ Department of Neonatology, Division of Paediatrics, University Medical Centre Ljubljana, Slovenia, ${ }^{3}$ Department of Pediatrics, Faculty of Medicine, University of Ljubljana, Ljubljana, Slovenia, ${ }^{4}$ Radiology Unit, Division of Paediatrics, University Medical Centre Ljubljana, Slovenia, ${ }^{5}$ Department of Urology, Division of Surgery, University Medical Centre Ljubljana, Slovenia, ${ }^{6}$ Department for Infectious Diseases, University Medical Centre Ljubljana, Slovenia

\section{Correspondence: \\ petja_fister@yahoo.com \\ Tel. 0038615229274 \\ Fax.: 0038615224035}

Received: March 28, 2019

Accepted: May 20, 2019

Key Words: Pyocele - Infection - Hydrocele - Streptococcus Pneumoniae - Neonate.
Objective - Bacterial infection of the scrotal appendages (scrotal pyocele) is a rare condition, especially in neonates. It is easy to confuse with other scrotal emergencies that require surgical intervention and orchiectomy. We present a case where pre- and intraoperative diagnosis was not clear and orchiectomy was performed; additionally, review of the literature, diagnostics, treatment options and lessons learned are discussed. Case report - A ten-day old full term neonate, born with bilateral hydroceles and glandular hypospadias, presented with a six day history of poor feeding and irritability; clinical examination revealed an enlarged, erythematous and tender right hemiscotum. Blood cultures, routine laboratory examinations and ultrasound (US) imaging was performed. Postnatal torsion with non-viable testicle, testicular malignancy and epididymo-orchitis were considered in the differential diagnosis. Upon surgical exploration, the testicle appeared non-viable due to severe inflammation and was removed. The patient was treated initially with ampicillin and gentamicin. After Streptococcus pneumoniae was cultured from specimens, treatment was continued with benzyl penicillin. Conclusion - Neonatal scrotal pyocele is an extremely rare condition. The main suggestive features are clinical presentation with signs of infection and an enlarged painful scrotum. US is the investigation of choice to differentiate from a surgical emergency. Free fluid in the scrotal cavity with septae, normal looking testicle on US and no signs of torsion at exploration are diagnostic signs. If diagnosed early, the first line of management is surgical aspiration or decompression, followed by broad-spectrum antibiotic treatment. With delay in diagnosis, the testicle may look non-viable and push surgeons for orchiectomy.

\section{Introduction}

Pyocele is an unusual urologic emergency, especially in the neonatal period, presenting as an acute scrotum. Due to the purulent collection within the potential space between the visceral and parietal tunica vaginalis around the testicle, it is also described as an infected hydrocele. It typically manifests with fever, tenderness of the scrotum or inguinal canal, erythema and sometimes scrotal discol- oration (1-4). Since 1975 only a few cases of neonatal pyocele have been described (1-11). Herein, we present a case of a septic ten-dayold neonate admitted to the Department of neonatology with an acute scrotum; postnatal testicular torsion with non-viable testicle, testicular malignancy or severe epididymoorchitis were suspected but the condition turned out to be a pyocele, caused by Streptococcus pneumoniae. 


\section{Case Report}

A ten-day-old boy was admitted to the Department of Neonatology with a six-day history of irritability, lack of appetite and poor weight gain. He was from a Romani family, born full-term and without significant perinatal history; at first clinical examination after birth, glandular hypospadia and bilateral hydroceles were found. At admission, he was febrile $\left(40^{\circ} \mathrm{C}\right.$, measured tympanically), tachypnoic and tachycardic, with a toxic appearance and prolonged capillary refill. Physical examination revealed a tense abdomen, sensitive to palpation and enlarged liver. Glandular hypospadia with cleft prepuce was present. The scrotum was bilaterally enlarged, with the right hemiscrotum being larger, erythematous, painful and tense on palpation. Both hemiscrota could be transilluminated appropriately.

After initial laboratory examinations, blood and urine cultures were taken, empiric antibiotic treatment with ampicillin and gentamicin was started. Laboratory findings suggested systemic infection with leucocytosis (WBC count $16.500 / \mathrm{mL}$ ), elevated C-reactive protein $(124 \mathrm{mg} / \mathrm{L})$ and procalcitonin $(1.38 \mathrm{~m} / \mathrm{L})$. Peripheral blood culture, urine culture and cerebrospinal fluid culture remained sterile. Because of tachypnea and oxygen requirement, a chest X-ray was performed, showing left pleural effusion
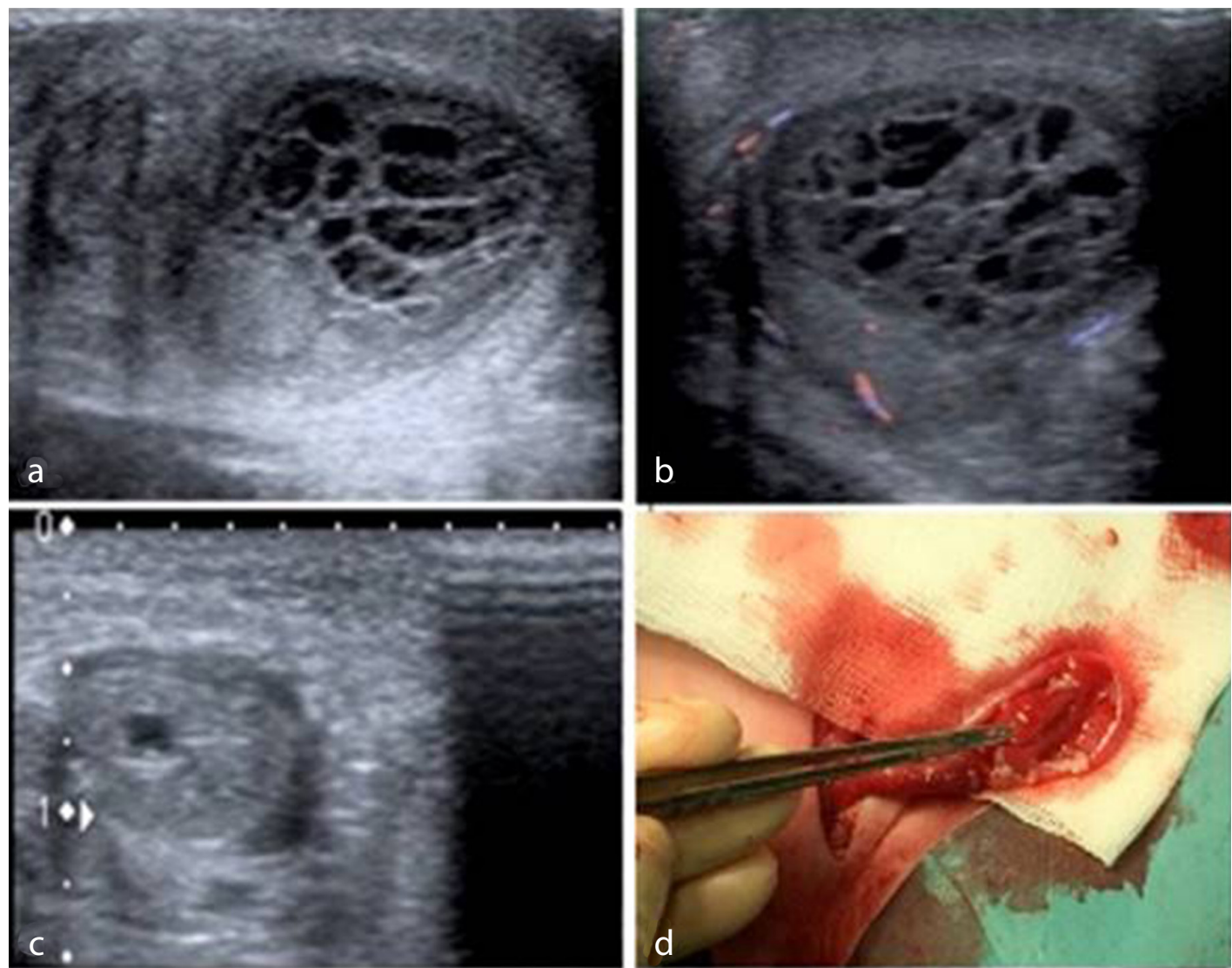

Fig. 1. The Scrotal Ultrasound Showed Thick Septated Fluid in the Right Hemiscrotum Transverse (A) and Longitudinally (B) with Absent Doppler Signals. Normal Left Testicle (C). The Explorative Surgery Revealed the Normal Sized Right Testicle with Inflamed and Thickened Adjacent Structures and Pus Collection in the Tunica Vaginalis (D). 
and diffuse interstitial infiltrates. Scrotal ultrasound (US) showed a normal sized right testicle with normal Doppler vascularity; the testicle was surrounded by thick, abundant, and uniformly septate fluid (Fig. 1). It was unclear whether the septate fluid represented the fluid in the scrotum, or the septate area was a part of a less vascularized tumour. The left testicle and abdominal organs had normal appearance on US.

Surgical exploration of the right hemiscrotum under general anaesthesia was performed. A normal sized right testicle, with no signs of torsion was seen, but inflamed and thickened adjacent structures and pus collection in the tunica vaginalis were found (Figure 1D). Because of severe inflammation, the testicle appeared macroscopically nonviable and semicastration was performed without deciding for performing frozen sections in the less possible malignant etiology. The postoperative course was uneventful. Systemic and local signs of inflammation subsided. Control chest X-ray demonstrated no pleural effusion with no infiltrates within lung parenchyma. Antibiotic treatment was continued.

Tissue and fluid samples taken during surgery were cultured. Streptococcus pneumoniae was successfully cultured on enriched media. After results of antibiotic susceptibility, treatment with benzyl penicillin was continued until the $7^{\text {th }}$ postoperative day.

Histologic findings revealed a pyocele of the right hemiscrotum with intact histological structure of right testicle and epididymis, tunica vaginalis coated with fibrin and numerous neutrophil granulocytes without histological evidence of tumour growth. The patient was followed-up by a urologist, for surgical correction of hypospadias.

\section{Discussion}

Prompt investigations and surgical exploration of scrotum in a clinically septic neonate with left pleural effusion, diffuse interstitial infiltrates, enlarged liver and increased inflammation parameters revealed that the cause of acute scrotum was a pyocele rather than testicular torsion, less vascularized tumour or severe epididymo-orchitis as it was clinically anticipated.

According to the literature, there are three mechanisms described in the pathogenesis of pyocele: haematogenous seeding of the serosa lining, secondary infection of a hydrocele following orchitis or epididymitis, or seeding from an intraperitoneal source through a patent peritoneal pouch (lat. processus vaginalis), as in acute appendicitis $(1,4,7)$. The main pathogens described in pyocele are Echerichia coli and Staphylococcus spp., but Klebsiella pneumoniae and other coliforms, $\beta$-hemolytic streptococci, Salmonella spp, Bacteroides fragilis and Proteus mirabilis have also been reported as etiological agents. Nonetheless, the majority of cases of pyocele are idiopathic in aetiology and the source of infection remains unrecognized (1, 3-6).

The infectious agent in our case was Streptococcus pneumoniae. The patient presented with clinical and laboratory signs of bacterial infection so we think that, despite sterile blood cultures, the mechanism of infection was haematogenous seeding of the serosa lining of the testicle. The differential diagnosis of acute scrotum in neonates is broad. Possible causes are testicular torsion, torsion of the spermatic cord, appendix of the testicle or epididymis, a poorly vascularized tumour, incarcerated inguinal hernia, acute scrotal idiopathic oedema, or acute epididymo-orchitis.

US with colour Doppler imaging has become the standard for evaluation of the acutely swollen scrotum (8). It can readily differentiate between normal and abnormal scrotal contents and allows assessment of viability in testicular torsion $(4,9)$. However, as previously reported in some cases of neonatal pyo- 
cele, US imaging can be misleading. Infantile pyocele and a scrotal abscess can mimic the Doppler sonographic findings in missed testicular torsion $(6,10,11)$. In our patient the testicle looked viable, homogenous, and normal Doppler flow within the testicle was visible. The free scrotal fluid with septations and debris seen on US is very suggestive of a scrotal pyocele. This and the fact, that no torsion of the spermatic cord was seen at exploration, might have helped the surgeon to preserve the testicle. It is understandable however, that the rarity of this condition, uncertainty of the imaging, general condition of the patient, and macroscopic appearance of the scrotum at exploration, made the surgeon decide towards orchiectomy.

Treatment of pyocele requires empirical broad-spectrum antibiotics with later adjustment according to antibiotic susceptibility testing performed on cultures of bacteria grown from surgical specimens. If the pyocele remains untreated, it can organize into a scrotal abscess with a distinct well-demarcated hyperaemic wall around the purulent fluid collection (4-6). The majority of published cases have been definitively treated with surgical drainage, however many patients ultimately require orchiectomy due to disease progression or its severity $(1,2,3,5,7)$. Percutaneous image-guided aspiration described by some authors is an effective management option for paediatric pyocele avoiding the risks of an invasive procedure, orchiectomy and general anaesthesia (1).

\section{Conclusion}

Pyocele is a rare urologic emergency in the neonatal period. Nevertheless, this clinical entity should be considered in the differential diagnosis of acute scrotum and fever in a neonate. The main suggestive features are clinical presentation with signs of infection, free fluid in the scrotum with septation, normal looking testicle on US, and no signs of torsion at exploration. If diagnosed early, the first line management is treatment with broad spectrum antibiotics and surgical aspiration or decompression. In severe advanced forms however, the testicle may look nonviable and push surgeons for orchiectomy. In the case presented the management could have been optimised by earlier recognition of the condition.

Authors' Contributions: Conception and design: TL and PF; Acquisition, analysis and interpretation of data: TL, DPP, MT and PF; Drafting the article: TL, PF; Revising the article critically for intellectual content: DPP, RK, LL and PF; Approved final version of the manuscript: TL, DPP, MT, RK, LL and PF.

Conflict of Interest: The authors declare that they have no conflict of interest.

\section{References}

1. Oberlin DT, Cheng EY. Management of pediatric pyocele using percutaneous imaging-guided aspiration. Int J Surg Case Rep. 2015;16:119-21.

2. Terentiev V, Dickman E, Zerzan J, Arroyo A. Idiopathic infant pyocele: a case report and review of the literature. J Emerg Med. 2015;48(4):93-6.

3. Chiang MC, Wang TM, Fu RH, Chu SM, Chou YH. Early-onset Escherichia coli sepsis presenting as acute scrotum in preterm infant. Urology. 2005;65(2):389.

4. Kim KK, Park HW. An unusual infected hydrocele-a case report. J Korean Med Sci. 1995;10(1):42-3.

5. Mutlu M, Imamoglu M. Scrotal Abscess in A Newborn: Caused by extended-spectrum betalactamase-producing Klebsiella pneumoniae. Indian J Pediatr. 2010;47:363-4.

6. Ezomike UO, Ituen MA, Ekpemo SC, Ekenze SO. Right paratesticular abscess mimicking neonatal testicular torsion and caused by Proteus mirabilis. Afr J Urol. 2013;19:202-4.

7. Kraft KH, Lambert SM, Snyder HM, Canning DA. Pyocele of the scrotum in the pediatric patient. J Pediatr Urol. 2012;8(5):504-8.

8. Avery LL, Scheinfeld MH. Imaging of penile and scrotal emergencies. Radiographics. 2013;33(3):721-40. 
9. Munden MM, Trautwein LM. Scrotal pathology in pediatrics with sonographic imaging. Curr Probl Diagn Radiol. 2000;29(6):185-205.

10. Lim GY, Lim SA, Jeong YJ, Hahn ST, Lee JM. Infantile scrotal pyocele simulating missed tes- ticular torsion on sonography. J Clin Ultrasound. 2003;31(2):116-8.

11. Raveenthiran V, Cenita S. Scrotal abscess mimicking testicular torsion in infants and neonates. J Pediatr Surg. 2007;42:597-8. 